Yam Bahadur Roka

MS, MCh., IFAANS

\author{
Mohan Karki \\ MS \\ Department of Neurosurgery \\ Neuro Cardio and Multispeciality Hospital \\ Jahada road, Birtatnagar-13, Nepal
}

Address for correspondence:

Dr. Yam Bahadur Roka

M.S. (Surgery), M.Ch. (Neurosurgery), F.N.N.I. (Skull

Base Surgery), IFAANS

Chief of Neurosurgery

Neuro Cardio and Multispeciality Hospital

Biratnagar, Nepal

Email: dryamroka@yahoo.com

Received: $1 / 11 / 2017$

Accepted: 6/1/2018

\section{Post Traumatic Chronic Encapsulated Intracerebral Hematoma in a Child: Case Report and Brief Review of Literature}

Chronic encapsulated intracerebral hematoma $(\mathrm{CE}-\mathrm{ICH})$ is an uncommon pathology that presents with headache, seizure, focal neurological deficits, or as a tumor.Trauma as a cause for $\mathrm{CE}-\mathrm{ICH}$ is even rare and we believe this is the first case report as "trauma causing chronic encapsulated intracerebral hematoma "search in PubMed did not reveal any results. Repeated micro-hemorrhages in the $\mathrm{CM}$ or AVM are supposed to cause this lesion which progress from an earlier encapsulated phase to a thick capsulated stage with edema and clinical symptoms.CT or MRI is the diagnostic modality and it mimics, tumor, AVM, CM, angiomableed, cerebral abscess, metastatic mass or neurocysticercosis.Burr hole, mini-craniotomy, craniotomy, CT guided stereotactic aspiration or endoscopic excision are some options with equally good results. The present case with history of trauma was managed successfully with craniotomy with no recurrence for past one year.

Key words: burr hole, craniotomy, chronic encapsulated intracerebral hematoma, VGEF, tumor

a child presented with history of chronic seizures and was diagnosed as a glioma.

deficits, or as a tumor. It was first described by Yashon and Kosnik in 1978 and later elaborated by Hirsch(1981) with his 2 cases. ${ }^{1,2} \mathrm{~A}$ present search for CE-ICH in PubMed showed only 57 results suggesting its rarity with the first report from South Asia only in $2011 .{ }^{3} \mathrm{CE}-\mathrm{ICH}$ is difficult to diagnose preoperatively where it is usually reported as tumor, abscess, metastatic lesion or parasitic infections. Burr hole, mini-craniotomy, craniotomy, CT guided stereotactic aspiration or endoscopic excision are some options with equally good results. We report a case where

\section{Case Report:}

An 8-year old female child presented with history of repeated uncontrolled seizure for the past three years. She was on multiple anti-epileptic medications with poor control of her symptom. For the past one month she had severe headache associated with vomiting, reduced appetite and progressively increasing weakness of her left half of her body. Further questioning revealed significant trauma to head, secondary to fall,one monthprior to the
\end{abstract}



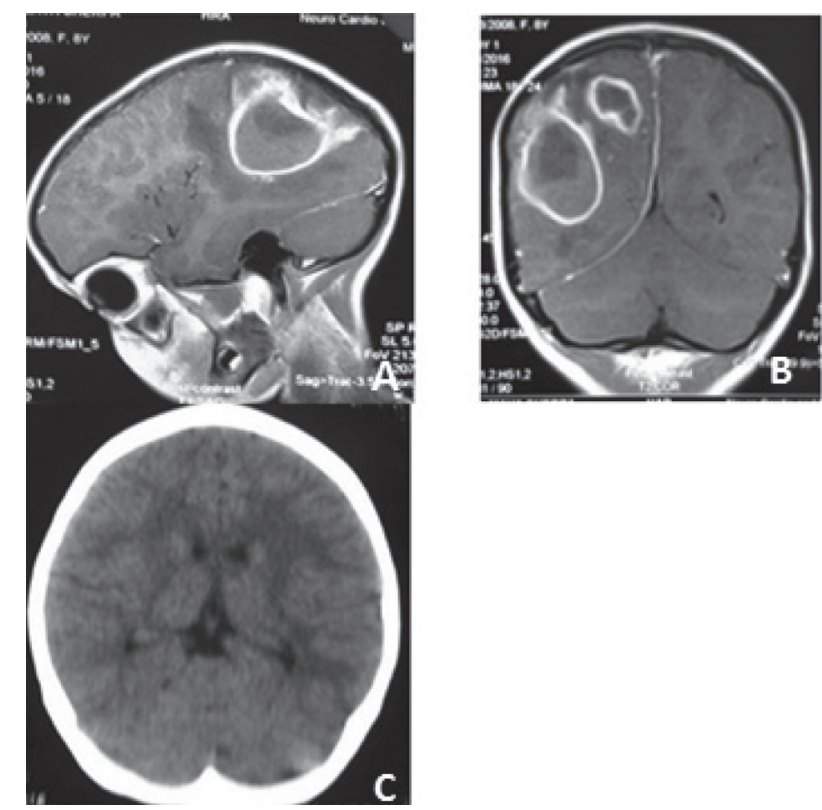

Figure 1: Preoperative contrast MRI sagittal and coronal (A,B)showing muliloculated right parietal lesion and complete excision on the postoperative CT scan (C).

onset of symptoms three years back. Clinical examination showed her to be conscious but irritable, early papilledema bilaterally and weakness of left half of 4/5 MRC grade. An urgent computed tomogram (CT) showed a large right temporo-parietal mass with peripheral ring enhancement and edema suggestive of cystic glioma. Magnetic Resonance Imaging (MRI) was done that revealed similar findings with a hypo intense center and hyper intense periphery on T1-weighted imaging and heterogeneous hypointensity on T2-weighted imaging with heterogeneous enhancement and thick walled capsule after gadolinium injection with surrounding edema (Figure 1A,B).

Right temporo-parietal craniotomy was performed which revealed a well-defined, thick walled lesion with heterogeneoushemorrhagic contents, clots, brown thick fluid with surrounding edema which was completely excised. No Vascular abnormality was detected. Postoperatively her weakness improved over the week and she was discharged on the $9^{\text {th }}$ day with antiepileptic. Follow-up scan did not reveal any recurrent or residual $\mathrm{CE}-\mathrm{ICH}$ and she is seizure free on mono-therapy for the past one year (Figure 1C). The histopathology showed the lesion as CE-ICH (Figure 2).

\section{Discussion:}

$\mathrm{CE}-\mathrm{ICH}$ is a rare entity that is usually associated with cavernous malformation (CM), arteriovenous malformation (AVM), alcoholic cirrhosis with coagulopathy and post radiosurgery forAVM. ${ }^{4,5}$ Trauma as a cause for $\mathrm{CE}-\mathrm{ICH}$ is even rare and we believe this is the first case report as "trauma causing chronic encapsulated intracerebral hematoma " search in PubMed did not reveal any results. Repeated micro-hemorrhages (7\% per patient-year) in the CM or AVM are supposed to cause this lesion which progress from an earlier encapsulated phase to a thick capsulated stage with edema and clinical symptoms. ${ }^{5,6}$ The thick capsule leads to neovascularization, thrombus reorganization leading to re-bleed and further expansion. Vascular endothelial growth factor (VGEF) (high concentrations of which has been found in such lesions) is one of the angiogenesis factor that encourages

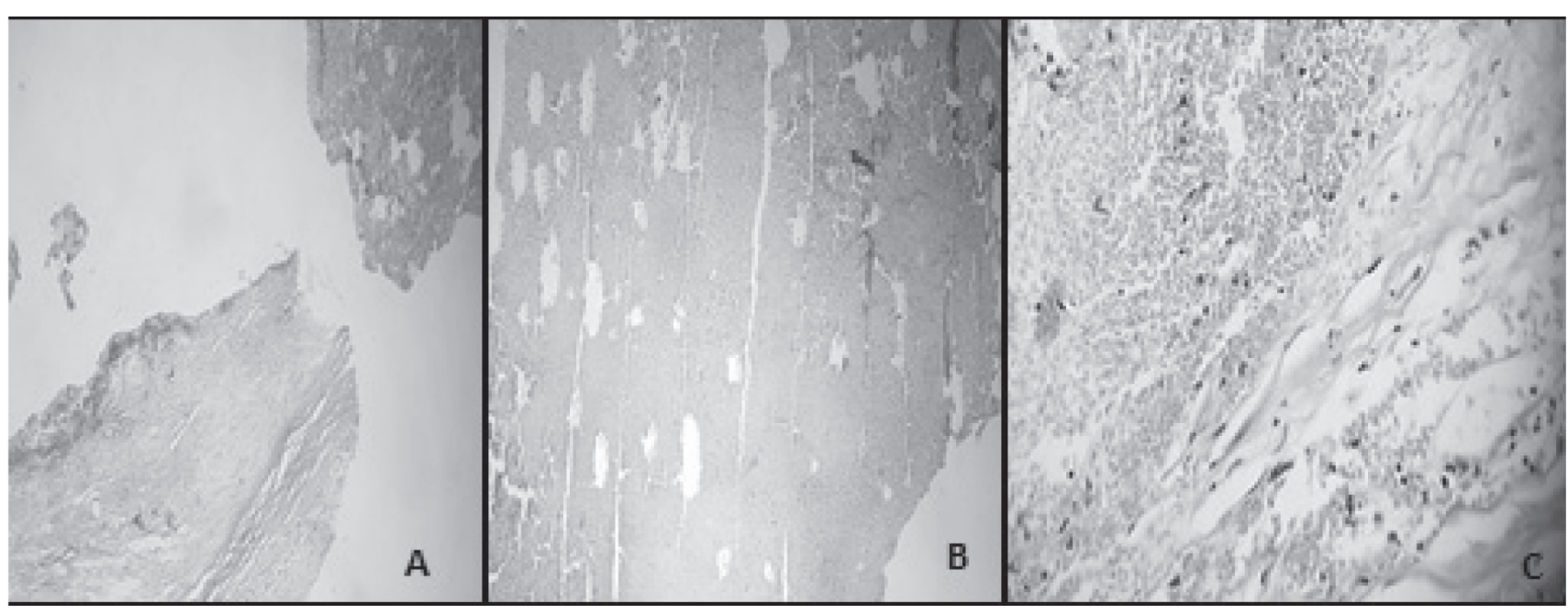

Figure 2.2A. (H\&E, x200hpf) left lower side showing the capsule comprising of collagenous layer with inner layer showing hemorrhages and deposits of hemosiderin. The right upper corner shows thin walled vascular channels with no interveninig brain parenchyma. 2 B. (H\&E, x 400 hpf) same as Fig A showing deposits of hemosiderin. 2C. (H\&E, x200 hpf showing hemorrhagic necrosis. 


\section{Roka et al.}

new vessel formation, endothelial growth, perifocal edema and increased vascular permeability. ${ }^{4,7}$

$\mathrm{CE}-\mathrm{ICH}$ presents with headache, seizure or neurological deficits which is chronic in nature. CT or MRI is the diagnostic modality and it mimics, tumor, AVM, CM, angioma- bleed, cerebral abscess, metastatic mass or neurocysticercosis. ${ }^{8,9}$ Arterial spin labeling or MR spectroscopy can be helpful to differentiate $\mathrm{CE}-\mathrm{ICH}$ from tumors. ${ }^{8}$ The radiological "layer sign" presenting as a fluid level adjacent to the clot and contrast extravasation following CT angiography ('the spot sign'), has been reported by some study to predict expansion in $\mathrm{ICH} \cdot{ }^{10,13}$ Preoperative diagnosis is almost impossible. Non-capsulated stage CE-ICH is managed conservatively and those with capsule need excision. Burr hole, minicraniotomy, craniotomy, CT guided stereotactic aspiration or endoscopic excision are some options with equally good results. ${ }^{11,12}$ Due to the large size the present case underwent craniotomy which is helpful to remove the lesion totally, the surrounding edema helps capsular dissection and to rule out any vascular lesion within or the periphery. Total removal is a must to prevent recurrences and we believe as the surrounding tissue is not invaded the neurological deficits improve post-surgery. Histopathology examinations will settle the diagnosis of $\mathrm{CE}-\mathrm{ICH}$.

\section{References:}

1. Backlund EO, von Holst H: Controlled subtotal evacuation of intracerebralhaematomas by stereotactic technique. SurgNeurol 9:99-101, 1978

2. Dowlatshahi D, Brouwers HB, Demchuk AM, Hill MD, Aviv RI, et al: Predicting Intracerebral Hemorrhage Growth With the Spot Sign: The Effect of Onset-to-Scan Time. Stroke 47(3):695-700, 2016

3. Fiumara E, Gambacorta M, D'Angelo V, Ferrara M, Corona C. Chronic encapsulated intracerebralhaematoma: pathogenetic and diagnostic considerations. J Neurol Neurosurg Psychiatry52(11): 1296-9, 1989
4. Ganapathy K. Chronic encapsulated intracerebral hematoma. Neurol India 59:504-5, 2011

5. Hsieh CT, Chang CF. Encapsulated chronic intracerebral hematoma mimicking neoplasm. ActaNeurol Taiwan18(3):227-8, 2009

6. Kamide T, Seki S, Suzuki K, Aoki T, Hirano K, Takahashi M, Nomura M. A chronic encapsulated intracerebral hematoma mimicking a brain tumor: Findings on arterial spin labeling of MRI. Neuroradiol J29(4):273-6, 2016

7. Shibao S, Akiyama T, Yoshida K, Akiyama T. Chronic Encapsulated Intracerebral Hematoma: Endoscopic Removal as Minimally Invasive Surgery for a Patient with Alcoholic Cirrhosis. NMC Case Rep J 4(2):5153, 2017

8. Shimizu K, Sadatomo T, Hara T, Ohba H, Yuki K, Kurisu K. Frequency and Predicting Factors on Chronic Expanding Intracerebral Hematoma in Spontaneous Intracerebral Hemorrhage. J Stroke Cerebrovasc Dis26 (7):1541-1546, 2017

9. Takei J, Tanaka T, Yamamoto Y, Teshigawara A, Tochigi S, Hasegawa Y, Murayama Y. Chronic Encapsulated Expanding Thalamic Hematoma Associated with Obstructive Hydrocephalus following Radiosurgery for a Cerebral Arteriovenous Malformation: A Case Report and Literature Review. Case Rep Neurol Med 2016:5130820, 2016

10. Takeuchi S, Takasato Y, Masaoka H. Chronic encapsulated intracerebralhaematoma formation after radiosurgery for cerebral arteriovenous malformation. Neurol India 59:624-6, 2011

11. Takeuchi S, Wada K, Sakakibara F, Mori K. Chronic encapsulated intracerebral hematoma associated with cavernous malformation. J Korean NeurosurgSoc 55(2):89-91, 2014

12. Takeuchi S, Y. Takasato, H. Masaoka et al., "Development of chronic encapsulated intracerebral hematoma after radiosurgery for a cerebral arteriovenous malformation," Acta Neurochirurgica151(11):1513-1515, 2009 